\title{
Study of microwave induced secondary structural evolution in Antheraea assamensis (muga) silk nanoparticles
}

\author{
Prithvi C. Asapur ${ }^{1}$, Santhosh Kumar Mahapatra ${ }^{2}$, and Indrani Banerjee ${ }^{1}$ \\ ${ }^{1}$ Central University of Gujarat \\ ${ }^{2}$ Central University of Punjab
}

August 23, 2020

\begin{abstract}
Silk Nanoparticles were synthesized using conventional desolvation and microwave-assisted radiolysis method from Muga silk fibroin. The secondary structure evolution due to two different synthesis methods was investigated using FTIR and Circular Dichroism (CD) spectroscopy. The microwave synthesized nanoparticles showed enhanced $\beta$-sheet content than desolvation synthesized nanoparticles. The conformational changes of Muga silk fibroin protein to nanoparticle followed an ordered transition from random coil to $\alpha$-helix then from $\alpha$-helix to $\beta$-turn and from $\beta$-turn to $\beta$-sheets where $\alpha$-helix and $\beta$-turn are the intermediate forms before getting stabilized to the metastable $\beta$-sheets structure. The thermodynamics involved in secondary structure evolution was studied from CD analysis and activation energy involved in the formation of the secondary structure was determined.
\end{abstract}

\section{Hosted file}

Research article_1.docx available at https://authorea.com/users/352395/articles/477147-studyof-microwave-induced-secondary-structural-evolution-in-antheraea-assamensis-muga-silknanoparticles 